Short Report

\title{
Challenges with targeted viral load testing for medical inpatients at Queen Elizabeth Central Hospital in Blantyre, Malawi
}

\author{
Alison Craik ${ }^{1}$, Priyanka Patel ${ }^{1}$, Pratiksha Patel ${ }^{1}$, Jane Mallewa ${ }^{1}$, Ken Malisita ${ }^{2}$, Joseph Bitilinyu-Bangoh ${ }^{3}$, \\ Joep J. van Oosterhout ${ }^{1,4}$, Christine Kelly ${ }^{5}$
}

1. Department of Medicine, College of Medicine, University of Malawi, Blantyre, Malawi
2. Ministry of Health, Lilongwe, Malawi
3. Central Laboratory, Queen Elizabeth Central Hospital, Blantyre, Malawi
4. Dignitas International, Zomba, Malawi
5. Malawi-Liverpool-Wellcome Trust Clinical Research Programme, Blantyre, Malawi

Correspondence: Dr Alison Craik (alisoncraik@ymail.com)

\section{Background}

\section{Abstract}

Approximately 75\% of medical inpatients at Queen Elizabeth Central Hospital (QECH) in Blantyre, Malawi are HIV seropositive, and a third of these patients are on antiretroviral therapy (ART). Malawi guidelines recommend targeted viral load (VL) testing for patients on ART for at least one year who report excellent adherence and present with a WHO clinical stage 3 or 4 HIV disease. A switch to secondline ART is only indicated if a VL result $>5000$ copies $/ \mathrm{mL}$ confirms treatment failure.

Methods

During an audit of targeted VL testing at QECH, all adult medical admissions were screened to identify those in need of VL testing. Daily review of inpatient notes ascertained whether VL testing was ordered and carried out. At 8 weeks post-discharge the laboratory database was checked for results and was triangulated with the HIV outpatient database to ascertain whether patients had attended clinic, received results, and if these results had been acted upon.

Results

Out of 81 patients recruited, $63(77 \%)$ had a VL requested. At 8 weeks post-discharge, nine patients (14\%) had VL results available. The median (IQR) waiting time for those with results was 29 days (20-47). Five patients had a VL > 5000 copies/mL. Of these patients, three attended clinic and one was switched to second-line ART. Of the remaining 55 patients awaiting results, the median (IQR) waiting time at the 8 -week follow-up point was 72 days (67-80). At 8 weeks post-discharge, 8 patients $(33 \%)$ had died.

Conclusions

Our findings demonstrate challenges with targeted VL testing at QECH. Only two-thirds of patients with clinical ART failure were identified as eligible for targeted VL testing, and of these less than one-sixth had VL results available after 8 weeks. Interventions such as point-of-care targeted VL testing could result in faster turnaround times. In the interim, we suggest further evaluation of the possibility of switching patients with clinical ART failure and a low CD4 count to second-line ART while awaiting VL results.

\section{Introduction}

The HIV seroprevalence among adult medical inpatients at Queen Elizabeth Central Hospital (QECH) in Blantyre, Malawi is approximately $75 \%$, and about one-third of the patients with HIV are on antiretroviral therapy (ART). ${ }^{1}$ The proportion of adult medical inpatients on ART increased from 25\% in 2013 to 28\% in 2014 and 31\% in 2015 (Peterson I, QECH electronic patient record data, 2016, personal communication). Malawi HIV guidelines recommend targeted viral load (VL) testing for patients who have been on ART for at least one year, present with a WHO stage 3 or 4 clinical event, and report good recent adherence to their ART regimen. A switch to a second-line ART regimen is only indicated after a VL result $>5000$ copies $/ \mathrm{mL}$ confirms ART failure. $^{2} \mathrm{QECH}$ is a tertiary referral hospital and processes batched dried blood spot (DBS) VL samples in its central laboratory. Inpatients requiring VL testing during admission are instructed to attend the outpatient HIV clinic 3 to 4 weeks later to obtain results. There are already significant challenges in linking HIV-infected patients to regular followup and ART. As the Malawian ART programme continues to develop, there is a greater risk that patients established on ART will present with ART treatment failure and that the current VL testing systems may not be robust enough to meet service demands to facilitate a timely switch of
ART. We therefore prospectively studied targeted VL testing among adult inpatients at QECH and report outcomes at 8 weeks post-discharge.

\section{Methods}

Ethical approval for this study was granted by the College of Medicine Research and Ethics Committee (COMREC). Over a 4-week period, all adult medical admissions were screened for the following eligibility criteria: patients on ART for at least one year and presenting with a WHO stage 3 or 4 clinical event, with self-reported excellent ART adherence (taken ART as prescribed over the previous one month). Written consent was obtained from each patient. Demographic information, duration on ART, and self-reported ART adherence data were collected through patient interviews and review of clinical files. Inpatient notes were reviewed daily to ascertain whether VL testing was ordered and carried out. At 3, 6, and 8 weeks following patient discharge, the laboratory VL database was checked for available results, which were triangulated with the HIV outpatient clinic electronic database to determine if patients had attended the HIV clinic, received their results, and were switched to second-line treatment (if indicated). Two attempts were made to contact patients via telephone, 8 weeks post-discharge, to obtain information on health status. 
Table 1: Summary of WHO stage 3 and 4 HIV diseases in the study population

WHO stage 3 or 4 condition

Tuberculosis Frequency

Community-acquired pneumonia 32

Non-typhoidal Salmonella sepsis 17

Bacterial meningitis 5

Disseminated Kaposi sarcoma

Cryptococcal meningitis

Oesophageal candidiasis

Sepsis (source unknown)

Other*

*Other: HIV encephalitis, HIV wasting syndrome, intracranial space occupying lesion (?lymphoma), lung abscess, E. coli sepsis, HIV-associated nephropathy, Pneumocystis jirovecii pneumonia

\section{Results}

Eighty-eight patients were screened and 85 patients consented to be enrolled in the study. Four patients were later excluded when their ultimate discharge diagnosis was no longer a WHO stage 3 or 4 condition. Data for 81 patients were included in the final analysis. The median (IQR) age was 37 years (31-43), and the median (IQR) duration on ART was 47 months (23-84). The median (IQR) CD4 count during the previous 6 months was 94.5 cells/uL (14.5-211). The most common clinical events were tuberculosis, communityacquired pneumonia, bacterial meningitis, and non-typhoidal

Figure 1: Intended viral load testing pathway at Queen Elizabeth Central Hospital
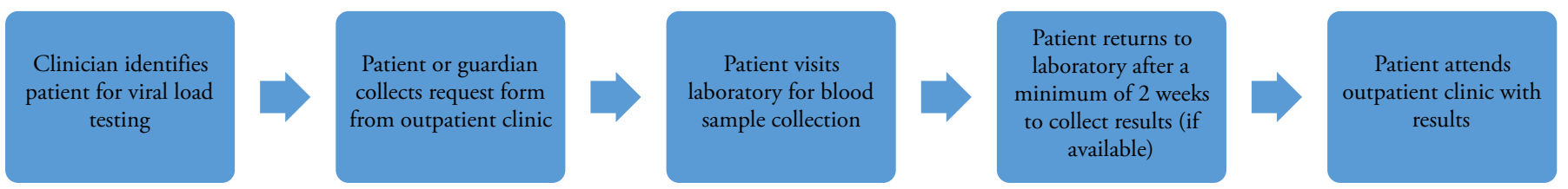

Figure 2: Viral load (VL) testing pathway for study patients including median waiting time for viral load results

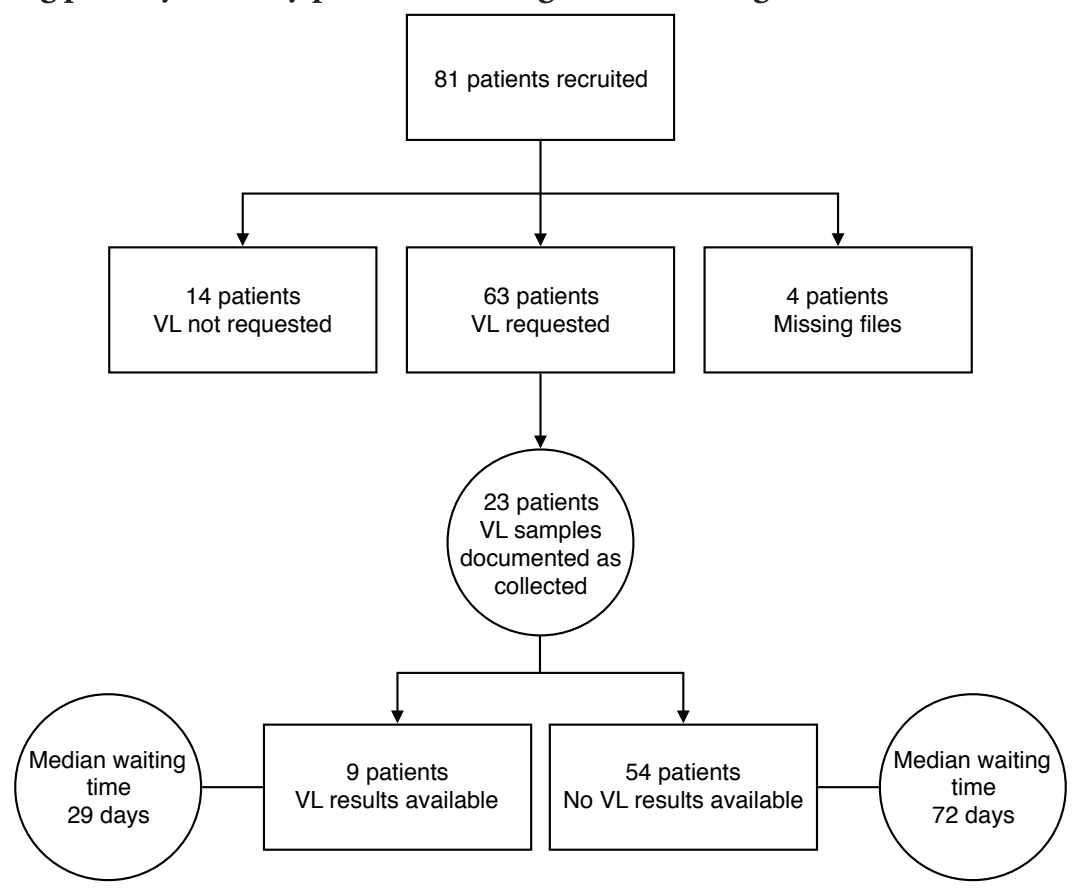


Figure 3: Patient outcomes at discharge and 8 weeks post-discharge

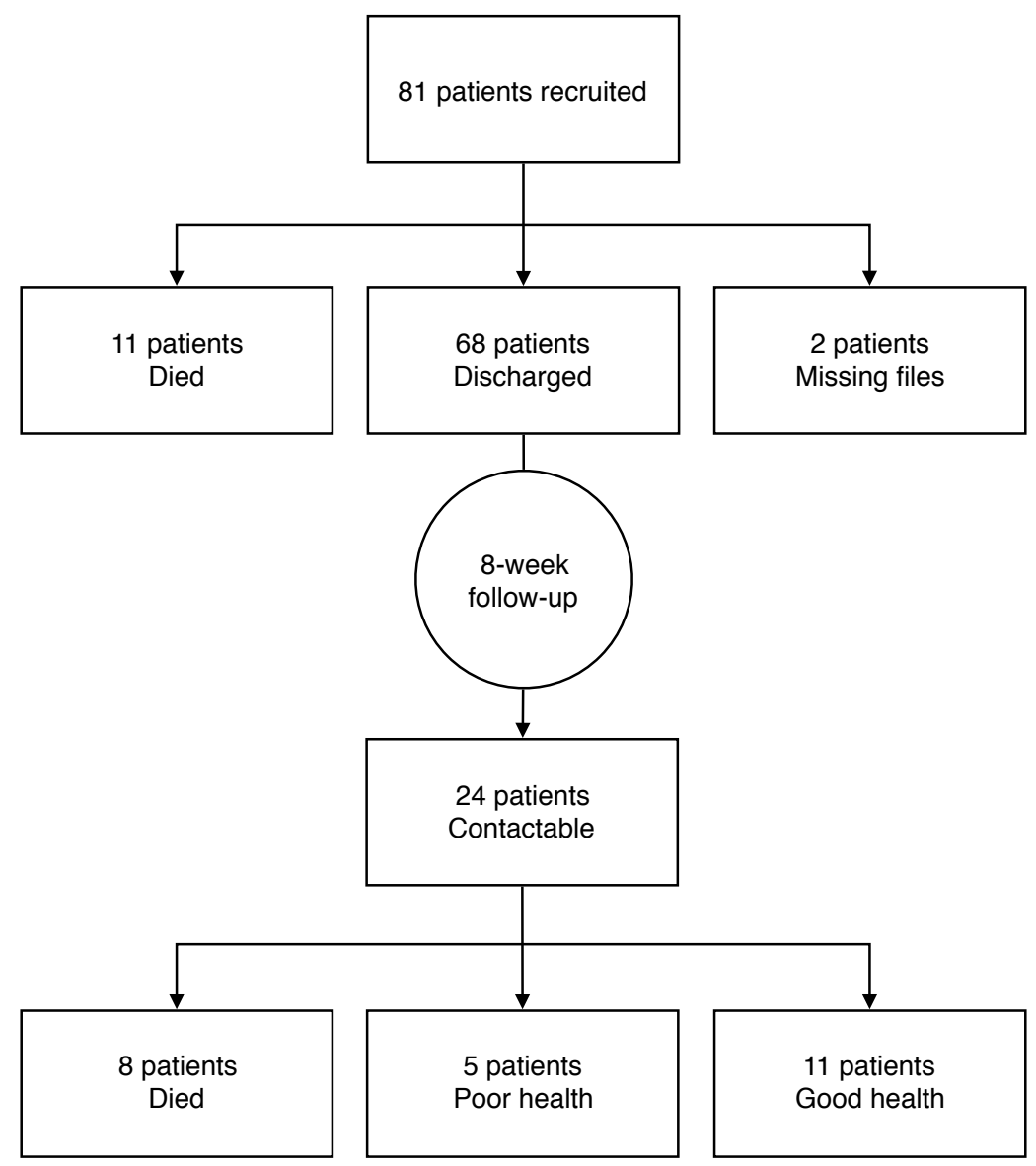

on average. Ultimately, only one patient was switched to a second-line regimen. The mortality rate in those who could be contacted was high.

The VL testing pathways at QECH are complex, with many steps required before a patient can receive results, as demonstrated in Figure 1. Delays are experienced throughout this process. Many patients were identified as needing a VL test, but because they were too sick and did not have relatives or guardians to escort them from the inpatient wards to the laboratory, these patients did not attend the laboratory for blood sampling,. Blood sampling is not carried out on the wards because of the need for dried blood spot testing and poor linkage of patients to their results when samples are obtained during their ward stays.

This was a single-centre experience, but we believe that our findings are likely to be generalisable to inpatients at other hospitals in Malawi, particularly those where there is no onsite testing. Apart from strengthening local systems, there are wider-scale interventions that could be considered. Using a point-of-care VL testing approach for patients with clinical failure would result in faster turnaround times by circumventing the need for batch testing. ${ }^{3}$ This could be of particular benefit for admitted patients who normally receive ART from community clinics a long distance from the referral hospital. In the interim, we suggest further evaluation of the possibility of switching a patient to second-line ART, while awaiting VL results, based on the presence of a clinical stage 4 opportunistic infection and a low CD 4 count. ${ }^{4,5}$ This would prevent long delays in effective ART for this high-risk patient group, who are often suffering from advanced ART failure, which exposes them to risk of further complications and death. ${ }^{6}$

\section{References}

1. SanJoaquin MA, Allain TJ, Molyneux ME, et al. Surveillance Programme of IN-patients and Epidemiology (SPINE): implementation of an electronic data collection tool within a large hospital in Malawi. PLoS Med. 2013;10(3):e1001400.

2. Ministry of Health, Malawi. Ministry of Health, Malawi. Clinical management of HIV in children and adults [Internet]. Lilongwe: Ministry of Health, Malawi; $2014 \mathrm{Feb}$ [cited $2016 \mathrm{Mar} 30] .95$ p. Available from: http://www.emtct-iatt.org/wp-content/uploads/2015/09/Malawi-HIVGuidelines-2014.pdf.

3. Stevens W, Gous N, Ford N, Scott LE. Feasibility of HIV point-ofcare tests for resource-limited settings: challenges and solutions. BMC Med. 2014 Sep 8;12:173.

4. Mugyenyi P, Walker AS, Hakim J, et al. Routine versus clinically driven laboratory monitoring of HIV antiretroviral therapy in Africa (DART): a randomised non-inferiority trial. Lancet. 2010 Jan 9;375(9709):123-31.

5. Waruru A, Muttai H, Ng'ang'a L et al. Positive Predictive Value of the WHO Clinical and Immunologic Criteria to Predict Viral Load Failure among Adults on First, or Second-Line Antiretroviral Therapy in Kenya. PLoS One. 2016 Jul 6;11(7):e0158881.

6. Paton NI, Kityo C, Hoppe A, et al. Assessment of second-line antiretroviral regimens for HIV therapy in Africa. N Engl J Med. 2014 Jul 17;371(3):234-47. 